\title{
VIGÍA, Observatorio Cultural de la Provincia de Cádiz \\ Un proyecto de apoyo al desarrollo cultural territorial
}

J. Luis Ben

Sanlúcar, Cádiz. Septiembre de 2004

Este es el texto de la conferencia ofrecida el martes 28 de septiembre en el marco del Curso de Planificación Estratégica de la Cultura (IX Cursos de Otoño de la UCA en Jerez)

El camino es largo, la vida breve y la verdad lejana.

Joseph Conrad

En primer lugar me gustaría señalar que el título de la ponencia resulta excesivo, quizás demasiado ambicioso para el tiempo del que disponemos. No se trata de que no se pueda definir con cierta claridad lo que es un observatorio cultural. El problema está en la segunda parte del enunciado, en el concepto de desarrollo cultural. Estamos ante un concepto complejo, muy trabajado a cualquier nivel y en el que la UNESCO lleva tiempo reflexionando. Para ser honesto con Uds. prefiero centrarme en describir lo que es un observatorio cultural, concretamente en VIGíA, antes que entrar en el jardín de tratar de aclarar lo que es el desarroIlo cultural. El jardín pudiera acabar en laberinto y no enterarnos de nada. Sin embargo para quienes tengan curiosidad en el tema no han de hacer más que el esfuerzo de dirigirse a la web de la UNESCO que es tan sencilla como www.unesco.org.

Entrando en materia lo primero es tratar de saber qué es un observatorio cultural. Si lo abordamos desde una perspectiva funcional, del para qué sirve, un observatorio no es más que una "herramienta para el conocimiento" de la realidad cultural de un territorio concreto. En nuestro caso de la provincia de Cádiz. Pero lo aparentemente sencillo no lo es en la realidad porque ża qué nos referimos al hablar de realidad cultural? ¿̇a la cultura creativa?, ¿̇a los aspectos sociales y educativos?, ¿hablamos de estética o quizás de antropología?, ¿̇nos centramos sólo en el arte?, ¿̇es la economía de la cultura lo que nos interesa? Lo dicho, de simple no tiene nada esta definición de salida. Otra precaución que hemos de tomar antes de profundizar en la materia es evitar el error de tratar de fijar un concepto de Cultura. Muchos han fracasa- do en el intento. Se me viene a la memoria una frase de San Agustín que afirmaba "¿Qué es el tiempo?" Cuando no me lo preguntan lo sé, pero cuando me lo preguntan ya no lo sé". Seamos prudentes y lleguemos a un cierto pacto. Todos intuimos qué es la cultura, no entremos en tratar de verbalizar una definición común y funcionemos con esa intuición genérica que planea sobre nuestras mentes. Seguro que nos evitamos problemas.

No obstante, sí hemos de tener en cuenta que la cultura no es una realidad monolítica, homogénea y que se nos presenta a todos bajo el mismo aspecto. Utilizando un adjetivo no demasiado hermoso podemos afirmar que la cultura es una realidad poliédrica, se muestra bajo muchas caras. De éstas, numerosas, existen algunas que nos interesan sobre las demás. Es evidente que no es posible abarcarlo todo, por lo que debemos ser selectivos. Destacaremos:

1. Los denominados "agentes culturales". Sin ser exhaustivos nos interesan los siguientes:

- Los responsables políticos. Ellos definen las políticas públicas, toman las decisiones.

- Los creadores. Escribió Eduard Delgado que los artistas son la materia prima de la cultura. Sobran los comentarios.

- Los profesionales. Gestores culturales y animadores socioculturales. Son los encargados de desarrollar y aplicar las políticas que definen los cargos públicos electos.

- Voluntarios. Gente, ciudadanos que se agrupan y reúnen para realizar o disfrutar de actividades culturales. Por ejemplo grupos de teatro aficionado, corales, guías, etc.

- Los públicos. Aquellos que deciden ir o no ir a un espectáculo o acto cultural. Quien dude que el público no es un agente cultural activo es que no se 
ha encontrado ante un patio de butacas vacío después de mucho trabajo.

2. Las "políticas culturales". Las grandes decisiones que se toman respecto a la intervención de las administraciones públicas en estos asuntos de la cultura. Se apoyan en la legitimidad democrática de los responsables electos y, en circunstancias óptimas, en la planificación estratégica territorial.

3. Los "proyectos", programas, acciones que realizamos como consecuencia de las políticas anteriormente definidas. Desde el punto de vista de la Gestión Cultural es imperativo poder evaluarlos, medirlos, conocer su validez.

4. El "impacto" de las acciones derivadas de las políticas culturales públicas. Es preciso conocer cómo políticas, agentes y proyectos influyen en lo cotidiano, en la vida de las gentes, en lo social, en lo económico, en lo estrictamente cultural, si es que llegamos a saber qué es eso.

Como remate de todo lo anterior podemos concluir que un observatorio deberá servirnos para conocer estos aspectos. Pero evidentemente ha de ser con un conocimiento real, científico si lo prefieren. Sin embargo hay un aspecto importante que me gustaría tratar, es la cuestión de por qué se crean los observatorios culturales. La respuesta es muy significativa para que podamos comprender mejor este tipo de instrumentos que son los observatorios. Afirmaba Sajarov, el físico y premio Nóbel ruso, "que tanto la ciencia como el arte nacen de la insatisfacción". Sólo aquellos hombres descontentos con las respuestas tradicionales se esfuerzan por encontrar nuevas soluciones y tanto da que se trate de arte como de ciencia. En este sentido el nacimiento de los observatorios culturales surge de una necesidad imperiosa de los profesionales del sector de la cultura. Una necesidad que tiene una doble faceta:

$1^{\circ}$. La necesidad de contar con información sistematizada y regular tanto en el tiempo como en las series.

$2^{\circ}$. La necesidad de conocer el verdadero impacto de las políticas culturales.

Lo realmente importante de esta doble necesidad que se halla en la génesis de los observatorios culturales es que condiciona sus funciones, como consecuencia de estas necesidades tenemos prefijados los dos grandes pilares sobre los que pivota la actividad de un observatorio cultural:

a) Investigación. Resulta asombroso que tras más de veinticinco años de democracia y políticas culturales las investigaciones resulten tan escasas, breves y desordenadas. Realmente hay hambre de investigación y de sus resultados entre los profesionales del sector.

b) Formación. Dada la desregulación que existe en las profesiones de la Gestión Cultural, su escasa validación social y la falta de conocimientos sobre las herramientas que aplicar en su trabajo, las carencias formativas de los gestores y de los responsables políticos inclusive, son enormes. Un observatorio cultural no puede ser ajeno a esta realidad.

Llega el momento de tratar de definir, a la manera clásica, sin incluir la palabra definida y en un párrafo breve. Para ello me permito un experimento lingǘstico un tanto heterodoxo. Si Uds. van a la página web de la UNESCO, la que les reseñé anteriormente, podrán encontrar un listado de observatorios culturales de todo el mundo. Se refieren al menos veintitrés de ellos, algunos muy peculiares. Al describir sus funciones, objetivos y contenidos se puede observar la repetición de algunas palabras. Me he permitido listar las más reiteradas. A saber:

1. Cooperación.

2. Investigación.

3. Desarrollo (territorial o cultural).

4. Política (cultural).

5. Información.

6. Planificación.

7. Evaluación.

Lo que haré en primer lugar será ordenar con un criterio meramente alfabético estas palabras. Nos quedaría de la siguiente forma:

1. Cooperación.

2. Desarrollo (territorial o cultural).

3. Evaluación.

4. Información.

5. Investigación.

6. Planificación.

7. Política (cultural).

Para llegar a nuestra definición de observatorio 
cultural lo que debemos hacer no es más que darle gramaticalidad a este listado de palabras añadiendo fundamentalmente algunos verbos. El resultado podría ser:

"Los observatorios culturales son organizaciones que tienen por objetivo fomentar la cooperación y el 'desarrollo cultural y territorial' mediante proyectos de 'evaluación', obtención de 'información e investigación' en torno a la 'planificación' de 'políticas culturales'".

Creo que hemos llegado a una definición aceptable de lo que es un observatorio. Pero podemos continuar nuestro experimento y tratar de obtener otra definición simplemente tomando nuestra lista de conceptos en el orden contrario. Nuestra nueva definición sería:

"Los observatorios culturales centran su actividad en las 'políticas culturales', su 'planificación' y realizan 'investigaciones' encaminadas a obtener la 'información' que nos permita la 'evaluación' de su influencia en el 'desarrollo cultural y territorial' en aras a mejorar las fórmulas de 'cooperación'".

En ambos casos el resultado es bastante satisfactorio y de una reflexión más pausada de ambas definiciones podríamos obtener la fijación correcta del concepto observatorio cultural. Reconozco que no hemos hecho más que un ejercicio de sofística pero ha de admitirse que el resultado no es malo y nos ha permitido comprender mejor lo que nos traemos entre manos.

Vayamos a nuestro caso concreto, a VIGíA, el observatorio cultural de la provincia de Cádiz. En primer lugar es preciso recordar el territorio en el que estamos, nuestra provincia. Un territorio que es el más meridional de la Unión Europea, con los mayores índices de paro y los niveles más bajos en los indicadores educativos, sociales o económicos. Desde el punto de vista geográfico y cultural Cádiz está fragmentada en al menos tres provincias: $\mathrm{Ba}$ hía de Cádiz, Jerez y su marco y, por último, el Campo de Gibraltar. Otras zonas como la Sierra, la Janda o el Bajo Guadalquivir se adscriben en mayor o menor medida a las anteriores. Esta heterogeneidad territorial es muy importante para nuestro proyecto, supone un reto analizar las propuestas y necesidades de una territorio tan complejo y por ello, además, tan rico.
Hemos de añadir que estos no son buenos tiempos para las macro organizaciones, para la puesta en marcha de grandes estructuras dotadas de presupuestos cuantiosos y numerosos recursos humanos. Los responsables políticos muchas veces ya tienen bastante con mantener en pie las actuales administraciones como para entrar en aumentar su número o tamaño. Por todo lo anterior estaba claro que nuestro proyecto debía nacer para servir a una realidad territorial compleja aunque rica y además, lo imponían las circunstancias, con unas dimensiones reducidas en lo orgánico. Si hubiéramos de sintetizar la génesis de VIGíA en pocos pasos, éstos serían:

$1^{\circ}$. Un pequeño núcleo de reflexión de gestores culturales preocupados por su profesión y sus problemas. Se concentran en torno a la revista Periférica que edita la Universidad de Cádiz y que en estos momentos es la única en España dedicada estos temas.

$2^{\circ}$. La idea esencial es que habría de tratarse de una estructura mínima pero que a la vez pudiera implicar al máximo de gente. Huir de fórmulas como los consorcios, fundaciones, institutos, etc. Huelen excesivamente a burocracia, a gasto.

$3^{\circ}$. Una institución "matriz" que acogiera el núcleo organizativo duro de VIGíA. En nuestro caso fue la Diputación de Cádiz que asumió políticamente el proyecto desde el primer momento y creyó en el mismo. Su aportación inicial fue la imprescindible para garantizar un funcionamiento de arranque: Un gestor cultural (el que les habla), un presupuesto de salida (20.000 euros) y los medios de gestión como son ordenador, teléfono, Internet, mesa, silla, etc.

$4^{\circ}$. Otras instituciones que mostraron desde el principio su afinidad e interés por el proyecto. Es el caso claramente de la UCA y más en concreto del Vicerrectorado de Extensión Universitaria que se identificaban con el observatorio tanto en el plano técnico como en su dirección política. Este vicerrectorado no era un novato en estas lides, sólo hay que recordar su puesta en marcha de investigaciones tan importantes como 2003 Estrategias en Puerto Real, ya finalizada, o las actuales de Algeciras o Sanlúcar.

Una tercera institución se unió al proyecto, desde el ámbito de lo financiero, fue la Caja San Fernan- 
do. Ésta supo ver los aspectos innovadores de VIGíA y realizó una aportación económica al mismo implicándose además en su dirección política y técnica.

En el plano puramente práctico, del funcionamiento y operatividad, este formato se organizaba de la siguiente forma:

a) Las instituciones implicadas firmaron "un convenio de cooperación" y colaboración específico para el observatorio. Se trata de algo tan simple como un contrato público en el que expresan su voluntad de aportar los medios y recursos indicados para poder desarrollar el proyecto.

b) Se contempla un nivel político de decisión. El "Consejo Director" y lo integran los representantes políticos electos de las instituciones: Vicerrectora, Vicepresidenta de la Fundación Provincial de Cultura y, designado por sus órganos correspondientes, el Jefe Provincial de Relaciones Institucionales de la Caja San Fernando. A ellos se añaden los más altos cargos técnicos el Director de Cultura de la Diputación y la Directora del Servicio de Extensión Cultural de la UCA. EL último miembro de este Consejo Político es el Director del Observatorio que actúa como secretario del mismo, con voz pero $\sin$ voto.

c) Nivel técnico de propuesta de actuaciones. El "Consejo Técnico" formado por el Director del observatorio, dos técnicos del Vicerrectorado de Extensión Universitaria, un técnico de Caja San Fernando, un técnico de la Fundación Provincial de Cultura y un técnico municipal de reconocido prestigio. Su misión es elaborar el programa y el presupuesto del observatorio para someterlo al criterio del Consejo de Dirección

Y nada más. Tan sólo estos órganos para dirigir el trabajo del observatorio. Se crea, con pocos pero escogidos elementos, una dinámica de proponer, estudiar, aprobar y ejecutar los proyectos de VIGíA.

El observatorio tiene unos objetivos de enunciado simple. "Obtener la información y los datos precisos para mejorar la gestión de las políiticas culturales que afecten al territorio provincial", es la finalidad esencial tal y como aparece recogida en las cláusulas del convenio de cooperación al que nos hemos referido arriba. Pero este enunciado simple se refleja en unas tareas complejas. Porque implica entrar a tratar de conocer esa realidad poliédrica que afirmábamos que es la cultura. $\bigcirc$ sea, investigar y obtener datos sobre los agentes culturales, los creadores, los sectores, los equipamientos, la economía de la cultura, el consumo cultural, los hábitos culturales, la producción de cultura, el desarrollo, etc. Es una tarea que se precisa hacer pero además, no de cualquier forma sino de manera ordenada y sistemática. La segunda parte será difundir estos datos e informaciones, primero a los interesados más directamente (responsables políticos, gestores, industrias culturales, creadores, etc.) y también a los ciudadanos en general.

Antes de entrar a detallar los proyectos que VIGíA desarrolla en la actualidad, he de aclarar algo de vital importancia. Se trata de un hecho que se encuentra en la base esencial del proyecto. El observatorio evalúa, diagnostica, estudia e investiga, en esencia trata de exponer la realidad. Pero VIGíA no actúa, esa es función de los gestores y de los creadores, ni tampoco decide, esa es una función intrínseca a los responsables políticos. Puntualizado lo anterior, que termina de acotar nuestra propuesta, veamos los proyectos concretos que VIGíA tiene en marcha.

A) Becas de Investigación sobre el sector de la Cultura en la Provincia de Cádiz. Se trata de un plan a tres años en el que se abren cuatro líneas de investigación sobre el hecho cultural. Estas líneas de trabajo son:

- Economía y cultura. En este primer año se va a medir el impacto económico de dos festivales de nuestra provincia.

- Públicos de la Cultura. En este 2004 la investigación se centra en el perfil de los usuarios de las bibliotecas rurales.

- Agentes culturales. Se va a investigar la estructura y dinámicas de los creadores del sector del Flamenco.

- Equipamientos culturales de proximidad. Nos referimos con esta denominación a las Casas de Cultura y a los Centros Cívicos. Se decidió prestar una especial atención a estos equipamientos dado que opinamos se trata de los menos definidos y dotados.

B) Sistema de Indicadores Culturales. La falta de estadísticas y de indicadores, cuantitativos y cualitativos, en el sector de la cultura es alarmante. Ca- 
recemos de referentes, de series temporales que nos vayan indicando la evolución del sector cultural en nuestra provincia. Tenemos en estos momentos trabajando a un equipo pluridisciplinar de la UCA en la elaboración de un conjunto de indicadores que nos permitan realizar esta tarea de medición periódica. Lo interesante es que hay contactos con otras diputaciones provinciales andaluzas interesadas en el proyecto. Si conseguimos incorporar a un número significativo de diputaciones tendremos el esqueleto del SIC de Andalucía en el ámbito de la cultura.

C) Formación. Más arriba comentábamos que los profesionales de la gestión cultural tienen carencias formativas amplias. Por otra parte estos mismos profesionales demandan formación para solucionar los problemas de gestión diaria a los que deben enfrentarse. En este programa contamos con el apoyo fundamental de uno de los socios de VIGíA, la universidad. De hecho este curso en el que estamos es un producto derivado de esta línea de actuación. Para fines de año realizaremos en colaboración con la Federación Andaluza de Municipios y Provincias (FAMP) un seminario sobre la recientemente aprobada Agenda 21 de la Cultura. Es una línea de trabajo que pensamos cuidar especialmente en los próximos años.

D) Publicaciones. Señalar que tan importante como investigar es publicar el resultado de las mismas. Por ello a principios del año próximo saldrán editadas las becas de investigación que se están desarrollando en la actualidad. Procuraremos dar una distribución eficaz a dichas publicaciones, que lleguen a las personas interesadas. Igualmente tenemos en fase de estudio dos publicaciones especializadas cara a su salida el próximo año.

E) Mapa de Equipamientos Culturales. Se trata del proyecto en el que personalmente tengo más ilusión. Primero porque estamos ensayando una metodología nueva para la elaboración de una cartografía cultural. Y en segundo lugar con el programa hemos abierto una línea de cooperación exterior en concreto con Marruecos. Es una acción que nos viene financiada por la Unión Europea (el programa Interreg III-A) a través de la Consejería de Cultura de la Junta de Andalucía. VIGíA presentó la propuesta a esta última administración que la aceptó plenamente.

Haciendo algo de historia es preciso señalar que la cartografía cultural no es algo nuevo en nuestro país, en 1989 aparecieron dos mapas culturales de las provincias de Sevilla y Barcelona. Ambos realizados por las respectivas Diputaciones. Siendo los dos buenos trabajos, el de Sevilla merece la calificación de excelente. Pero curiosamente no ha habido una continuidad en el tiempo y así Sevilla no ha vuelto a realizar otro mapa hasta el año pasado y con una metodología y unos resultados francamente malos, muy por debajo del año 89. Un mapa de equipamientos es ante todo una necesidad. Es una demanda clara si se pretende realizar una planificación territorial de las políticas culturales. Se precisa saber dónde y cómo ubicar los distintos tipos de equipamientos. Además, en nuestro caso concreto la construcción del mapa conlleva un proceso de evaluación paralelo que nos indique el estado de conservación y de uso de nuestros equipamientos culturales.

Pero la cuestión metodológica es clave en nuestra cartografía. Existen tres posibles maneras de encarar la realización de un mapa. La primera, muy habitual en este tipo de trabajos técnicos, es realizar un buen pliego de condiciones y sacar a concurso entre tres o más empresas privadas tipo consultoría. La empresa adjudicataria, bajo nuestra supervisión, es seguro que realizaría un mapa y que lo haría bien. Al final del proceso tendríamos un producto, y nada más. La segunda fórmula sería encargar el mapa a un grupo de funcionarios técnicos que seguramente funcionarían en un "tempo" funcionarial y desde perspectiva puramente administrativa. Puede que al final tuviéramos un mapa y con suerte que no fuera demasiado malo ni estuviera ya desfasado al presentarse. Existe una tercera opción, que es la nuestra, iniciar todo un proceso en el que primero aprendiéramos a construir un mapa cultural, convocando a quienes trabajarán en el mismo, a expertos y docentes en los numerosos aspectos que deberán tocarse: geógrafos, arquitectos, gestores culturales, metodólogos en investigación, informáticos, fotógrafos, historiadores del arte, gestores teatrales, etc. Un proceso formativo que nos daría al final la herramienta metodológica para obtener todos los datos precisos en la construcción del mapa. Al final lo que esperamos obtener son dos cosas: El Mapa de Equipamientos Culturales y Conocimiento. Esto último es probablemente tan importante como el mapa en sí mismo.

El segundo aspecto que hace interesante este programa es la cooperación con Marruecos, en concreto con la provincia de Tetuán. Puede existir la tentación de considerar esta cooperación como 
accesoria, como un peaje que pagar a cambio de obtener las muy cuantiosas ayudas europeas. $\mathrm{Na}$ da más alejado del ánimo de quienes estamos en el proyecto de VIGíA. Tan importante, o más si cabe, es que Tetuán tenga un buen mapa como que lo tenga Cádiz. Nuestros socios también aplican políticas públicas, también tienen necesidad de herramientas de planificación, también se merecen un buen trabajo y aprender con nosotros cómo se construye una cartografía cultural. Personalmente creo que estamos ante un reto y ante la posibilidad del Sur siendo pionero y dando una lección al Norte. Se trata de una oportunidad que no podemos desperdiciar.

Hasta aquí casi todo lo que quería decirles. Espero que se hayan enterado de qué cosa es un observatorio cultural y cómo va a trabajar el de nuestra provincia. El balance de estos primeros meses es realmente muy positivo. Sin embargo no hay que dormirse en los laureles. Me permitiré una cierta nota erudita para acabar. El escritor inglés Ciryl Collony escribió entre los años 1941 y 1942 un extraño y profundo libro titula- do El sepulcro sin sosiego, en el mismo reafirma su fe en toda la cultura europea, en la propia inglesa, en la francesa y, he aquí lo extraordinario, en la cultura alemana en esa nación que en esos momentos estaba bombardeando su propio hogar. Para Collony las bombas nazis no invalidaban el imponente valor de todo lo que Alemania había dado a Europa. Utiliza este inglés culto un símil para explicar la locura de esos años de guerra. Relata como Palinuro, el mejor piloto de Eneas, que conducía a los exiliados troyanos por las aguas del Mediterráneo, se queda dormido al timón de la nave y cae al mar. Un exceso de confianza lo hizo morir abandonado en una playa solitaria'. Para Collony éste ha sido el mal de la cultura europea, el exceso de confianza que la ha conducido al enfrentamiento que en esos momentos desgarra al continente. Ya sólo manifestarles que procuraré ser un piloto más despierto que el desgraciado Palinuro. Gracias.

1. O nimium caelo et pelago confise sereno/ nudus in ignota, Palinure, iacebis harena. Virgilio. Eneida $\mathrm{V}$, pp. 870-871. 